\title{
The Materials Genome Initiative: One year on
}

A little over a year has passed since US President Barack Obama launched the Materials Genome Initiative (MGI) to enhance the United States' global competitiveness by cutting in half the current time and cost of bringing new materials from the laboratory to the marketplace. MGI is part of the president's larger innovation and competitiveness agenda addressing advanced domestic manufacturing.

Traditional materials development can be described as a continuum that spans discovery to deployment and commonly takes 10 to 20 years to traverse. Thus, materials crucial to solving some of society's most pressing problems may have already been invented and are awaiting implementation in a manufactured product. MGI aims to accelerate this timeline by creating a materials innovation infrastructure (MII) that will more closely integrate experimental tools, computational tools, and digital data. Through the MII, the discovery-todevelopment continuum would become more iterative and collaborative, which the administration hopes will result in more new materials brought to market in a shorter amount of time.

In many ways, MGI is the next step in the natural evolution of a movement that began in the 1980s with accelerated advances in computation and the concept of "materials by design," and has continued through more recent activities in integrated computational materials engineering. According to Linda Horton, director of the Materials Sciences and Engineering Division at the Department of Energy (DOE), "The community is ready for this initiative. The confluence of computational and experimental advances has made the research community very well prepared for launching this activity."

Although the community may have the technological preparation to take on the challenges of MGI, fully embracing the initiative requires a cultural shift. "We're asking people to change the way they think about their work," said Jim Warren, special advisor to the director on MGI at the National Institute of Standards and Technology (NIST). "Make it, test it, make it, test it' is not an MGI approach. Experiments need to be carefully selected against models so one can enhance the value of the experiment and the model." This means bringing together the separate and often disparate communities of materials experimentation, simulation, modeling, and theory to form collaborative research networks.

As MGI develops, additional issues will have to be addressed, such as how to recognize and assign value to contributed data sets, and how to navigate and police the emerging world of open access.

Several government agencies are working to advance the initiative, coordinated by an interagency subcommittee chaired by Cyrus Wadia, assistant director for Clean Energy and Materials Research and Development at the White House Office of Science and Technology Policy. In FY 2012, DOE, NIST, the National Science Foundation (NSF), and the Department of Defense (DoD) have together invested over $\$ 60$ million in direct MGI activity and programs influenced by MGI, and the president has requested significant budget increases for FY 2013 to continue building on this progress.

NSF's Designing Materials to Revolutionize and Engineer our Future (DMREF) program supports research that would accelerate materials discovery and development by enabling a materials-by-design approach, whereby materials functions and properties could be predicted from first principles. Fundamental advances in materials understanding across length and time scales will allow the interrelationships between constitution, processing, structure, properties, performance, and process control to be established. DOE's Office of Science's Predictive Materials Science and Chemistry program has a similar approach, seeking to advance materials and chemical processes that will specifically address energy-related basic research challenges through computation, experiment, and data. NSF plans to award \$11 million in grants this fiscal year, while DOE plans to award up to $\$ 18$ million. Both agencies intend to continue and expand these programs, with NSF requesting more than \$30 million for FY 2013 and DOE's Office of Science requesting $\$ 20$ million.

DOE's Office of Energy Efficiency and Renewable Energy has its own set of activities to promote MGI, including a \$14 million "lightweighting" program (spread over FY 2012 and FY 2013) that aims to improve vehicle fuel efficiency by incorporating advanced materials that will be developed with the aid of predictive modeling.

DoD's investment in MGI covers the entire materials development continuum and aims to fund research that takes advantage of new approaches to modeling

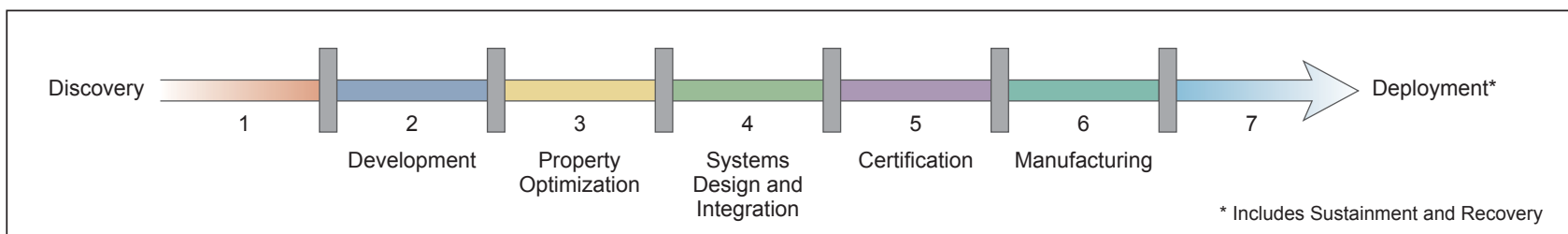

Traditional materials development commonly takes 10-20 years to traverse the discovery-to-deployment continuum. 
materials characteristics that will improve the prediction and optimization of materials properties for applications including body and vehicle armor, jet engines, and maritime and aerospace structures. These efforts are integrated into the activities of the Office of Naval Research, Army Research Laboratory, and Air Force Research Laboratory, including through the Multidisciplinary University Research Initiative, University Centers of Excellence, research contracts with industry, and Collaborative Research Alliance programs. DoD's FY 2012 investment in MGI is estimated at $\$ 17.3$ million, with a significant increase in funding planned in FY 2013.

NIST's MGI-related effort, Advanced Materials for Industry, aims to develop techniques and tools that will enable materials data, from both simulation and experiment, and modeling systems, operating over a range of length and time scales, to be more interoperable. The agency will also develop the means and standards for quality assessment of these models and the data generated from them. For FY 2013, NIST has requested an additional $\$ 10$ million for this program, which would bring its total annual investment to $\$ 14$ million.

It is important to keep in mind that MGI is more than a program; it is a scientific and cultural movement. As such, each agency is aligning and coordinating its investments with other initiatives. For example, both NSF and DOE's Office

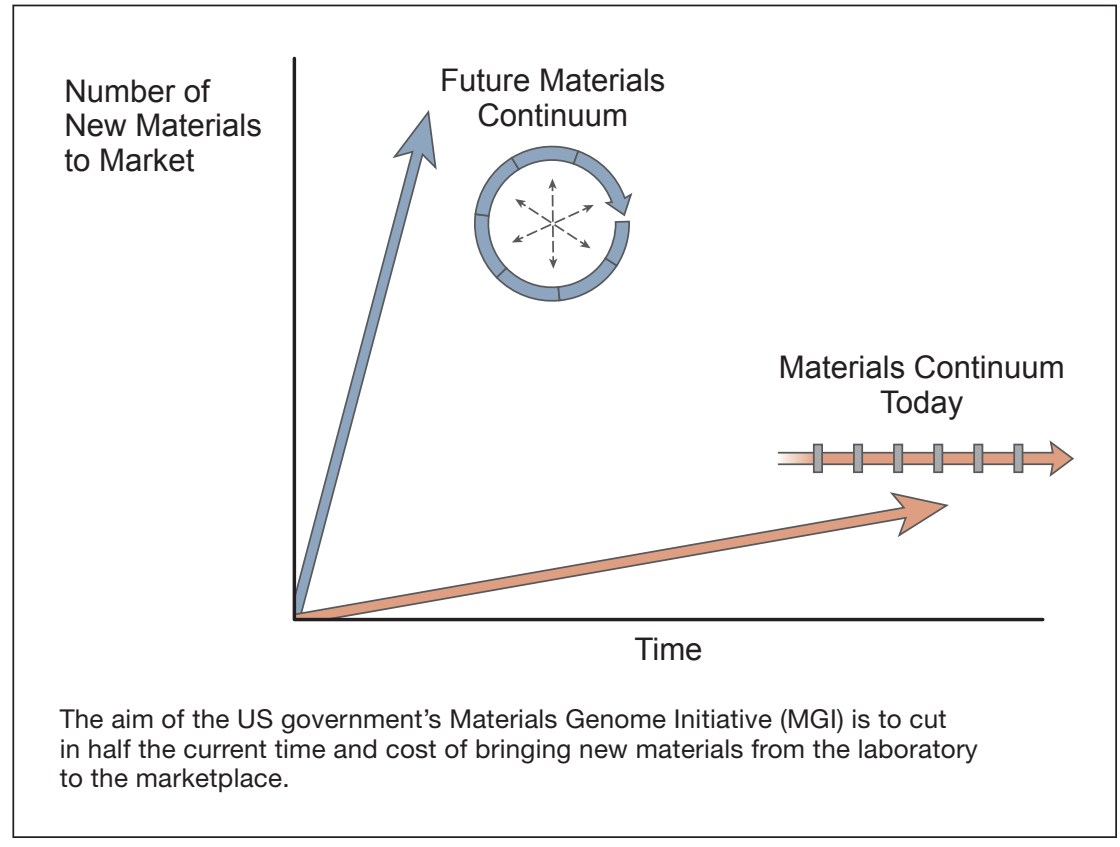

of Science are leveraging related initiatives at their agencies, such as Cyberinfrastructure for the 21st Century (CIF21) at NSF, and Scientific Discovery through Advanced Computing (SciDAC) and Computational Materials and Chemical Sciences Network at DOE. There are also strong ties to two other interagency administration priorities in advanced manufacturing and big data (see the July 2012 issue of MRS Bulletin, p. 628, for more on the Big Data R\&D Initiative).

Meeting the scientific, technologi$\mathrm{cal}$, and cultural challenges MGI presents will require the active participation of stakeholders in government, industry, and academia — an "all hands on deck" approach. Luckily, the initiative continues to gain support. In May, more than 60 companies and universities announced new commitments to MGI, joining a list of existing support from industry, academia, national laboratories, and professional societies.

Ashley White

AAAS Science \& Technology Policy Fellow

National Science Foundation

Any opinion, finding, and conclusions or recommendations expressed in this material are those of the author and do not necessarily reflect the views of the National Science Foundation.

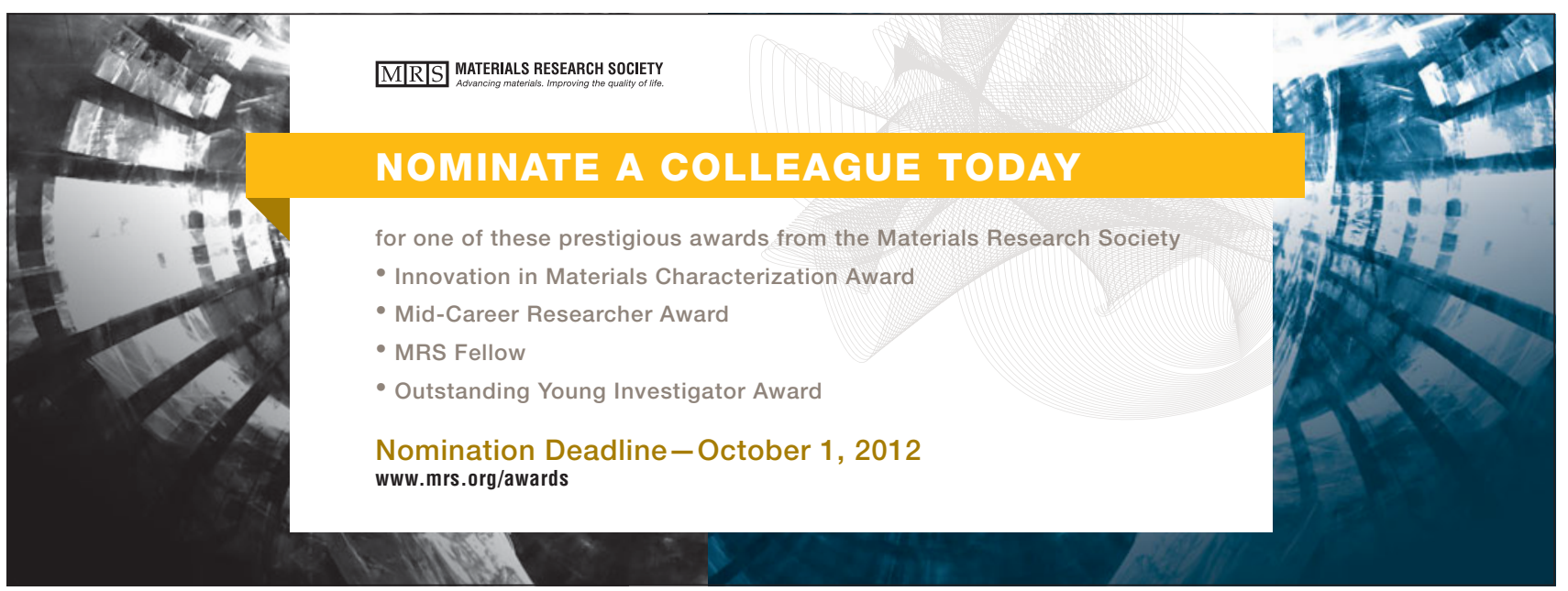

